\title{
State of the art of soft robotic applications based on magneto-rheological materials
}

\author{
Denys Gutenko ${ }^{1, *}$ \\ ${ }^{1}$ Cracow University of Technology, Warszawska 24, 31-155 Cracow, Poland
}

\begin{abstract}
Soft robotics is a new and exciting field of robotics which heavily relies on compliant materials. Soft robots attempt to copy the motion of living organisms and their adaptation mechanisms to the environment. New efforts in this field have paved way for the use of specific materials, e.g. electroactive polymers and/or magnetoactive (magnetorheological) MR elastomers. In this paper the author discusses the fundamental characteristics of MR fluids and elastomers, that allow them to be used as elements/components of soft robots. The advantages of using soft robots and the scope of applications for such robots are presented. The advantages and disadvantages of using MR fluids and elastomers in such soft robots are also considered. The history of the development of the idea of using MR fluids and elastomers in soft robots is presented, too. Possible applications for soft robots based on MR fluids and elastomers are considered. Various solutions for constructing a robotic gripper using MR fluids and elastomers are presented. Based on the above solutions, an analysis is made of the development of such technologies and the main problems are identified that will be the goal of solving them in the near future.
\end{abstract}

\section{Introduction}

Soft robotics is a field of robotics that relies on copying the movement of living organisms and the mechanisms of their adaptation to the environment and largely depends on compatible materials [1-2]. With the increase in the number of new technical solutions, the emergence of new materials and new urgent tasks, soft robots are finding even larger areas of application. This is also due to the fact that in some cases soft robots have revealed a number of advantages compared to rigid robots.

Nowadays, various approaches are used to build soft robots, such as shape memory alloys (SMAs) [3], pneumatic muscles [4-5], dielectric elastomers [6], magnetorheological (MR) materials [7], etc. Each of these approaches has its own advantages and disadvantages and its potential application scope. With the increasing diversity of the MR materials, there have appeared new opportunities for using these materials in soft robots. In this article, the author will consider the structures and properties of MR materials that allow them to be used in soft robots. Among materials other than MR foam [8], MR fluids and MR elastomers can be distinguished. These materials have properties that allow them to be used

* Corresponding author: denys.gutenko@doktorant.pk.edu.pl 
in various controllable application areas. Depending on the composition used, the characteristics of the obtained MR materials may differ, which allows them to perform various functions in soft robots.

\section{The structure and properties of MR materials}

MR fluid is a suspension of micron-sized iron particles in a non-magnetizable carrier fluid (oil or water) and additives [9-10]. The materials when exposed to magnetic field reveal a yield stress. MR fluids are known to operate in three fundamental modes: flow mode, shear mode and squeeze mode [11]. Their main application field is vibration damping.

For comparison, MR elastomers have two important elements: non-magnetic elastic matrices and magnetic particles [12-13]. Depending on the ingredients used, their proportions and manufacturing methods, various stiffness levels, saturation, hysteresis, and the reaction rate of the change in the property of the material to changes in the magnetic field can be obtained. Using specific manufacturing methods, the MR elastomers can be made isotropic or anisotropic. During the manufacture of the anisotropic MR elastomer at a certain period of time, the material is exposed to magnetic fields, as a result of which the magnetically active parts are aligned in chains along the magnetic field, which greatly affects the characteristics of the final product. In the isotropic MR elastomer, the magnetic particles are randomly arranged. As with MR fluids, there are three operating modes for the MR elastomer, two common for the MR fluid and the MR elastomer: shear, tension / compression and field active mode [14], the latter being useful in soft robots.

\section{Application of MR materials in soft robots}

\subsection{Absorbers, Sensors, Adhesion}

Due to their characteristics MR materials can be used in absorbers and isolators in different areas. MR fluids are used in dampers [15-16], rotary brakes and clutch systems [17], sandwich structures for vibration control of flexible structures [18], etc. On the other hand, MR elastomer applications can be seen in vibration isolators [19], dampers [20], etc. Also, there is evidence of MR elastomers being used as force/strain sensors [21-23]. One novel application area of MR fluids and MR elastomers is a solution for changing the adhesion of a material under the influence of a magnetic field. A chip transporter using MR elastomers is proposed in [24]. MR fluids also can be used as part of soft gripper due to their ability to change adhesion characteristics under the influence of magnetic fields [25].

\subsection{Stiffness change}

Depending on the property of MR materials used, these materials can act as various elements of a soft robot [7, 24-30]. In [28], a gripper based on MR fluid is proposed, which is capable of holding objects of various shapes. Holding force depends on the applied force and the shape of the object. As can be seen from the presented example, the change in the MR fluid's yield stress can be used to maintain an object at a fixed position. In this case, that state can be achieved under the influence of external forces or other soft robot actuators, which do not have to be based on MR materials. In order to use MR fluids in soft robots with many degrees of freedom, low power actuators can be used in combination with the fluid being under the influence of a magnetic field. One example of such a device is the snake-like robot in which MR fluid is inside the tube (see fig. 1 a). The position of the tube is altered using the low-power actuators when there is no magnetic field. When the desired 
position is reached, the magnetic field is turned on and the MR fluid develops a yield stress. The disadvantage of this approach is a complicated control system to handle both the actuators and the MR fluid's rheology.

Another example is a robot that can change its shape depending on what control signals were applied to low-power actuators located on its surface (fig. $1 \mathrm{~b}$ ). The fluid is inside the robot. When the desired shape is reached, a magnetic field is applied and the solidified MR fluid allows for maintaining its position. Such a robot has a potential to be used in applications from grippers to information visualizers. Again, the disadvantage of this approach would be its complicated control system.

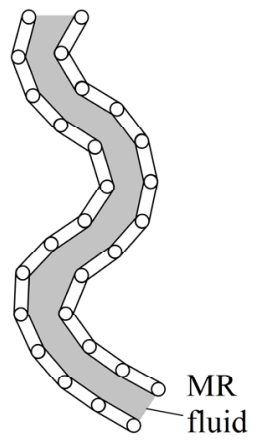

a)

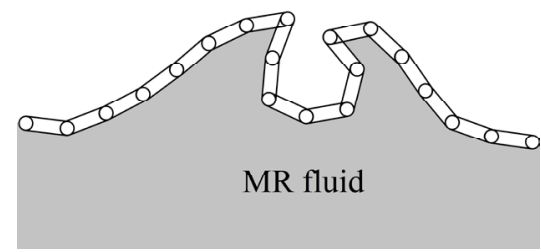

b)

Fig. 1. Examples of using MR fluid in soft robots: a) a snake-like robot using MR fluid, b) a gripper using MR fluid.

Next, one approach which is used in soft robotics relies on stiffness change [31]. MR elastomers operating in the field active mode can be also used in soft robotics [7, 29]. In this case, the desired state of the soft robot is the result of an equilibrium state in which the stiffness of the MR elastomer is compensated for by other forces. Any movement occurs in the absence of such equilibrium state until the soft robot reaches the desired equilibrium state. Thus, by changing the stiffness of the MR elastomer using a magnetic field, equilibrium position can be changed and therefore the position of the robot can be controlled. This approach may have several disadvantages, depending on what forces compensate the stiffness force of the MR elastomer.

If the stiffness force is compensated for by gravity, then given the constant direction of gravity forces, the area of work of the soft robot and, as a result, its application will be significantly reduced. An example is a piston that changes its height under the influence of a magnetic field (fig. 2 a). Inside the piston is an MR elastomer. In the absence of a magnetic field, the stiffness value of the MR elastomer is at a minimum value, the stiffness force is compensated by weight, and the piston height is minimal. Under the influence of a magnetic field, the stiffness of the MR elastomer increases. To compensate for the increasing stiffness force, the MR elastomer is extended until the stiffness force decreases to the level, where it can be compensated by the force of gravity. As can be seen from this example, such a piston can only work in an upright position, which, as was written earlier, limits its scope.

If the stiffness force of the MR elastomer is compensated by the stiffness force of the spring with a constant stiffness ratio, then, unlike the previous case, a soft robot can work in different positions, but this approach has its drawbacks, too. First, in addition to the stiffness force of the MR elastomer, the stiffness force of the spring in the opposite direction will always be present. So, in order to achieve the necessary resulting force of a soft robot, it is necessary to apply more force to compensate for the spring stiffness force. In addition, the maximum resulting strength of the soft robot may be different depending on 
the direction. In one direction, it can be limited by the maximum stiffness value of the MR elastomer, and in the other, by a constant value of spring stiffness. As an example, in fig. 2 b) a gripper is represented that contains an elastomer and a spring. Both the spring and the MR elastomer are in tension state. By changing the stiffness of the MR elastomer using a magnetic field, the position of the beam (which is located in the middle) can be changed and the object can be captured using both the left and the right parts of the gripper. Capturing and holding an object on the left side is carried out using MR elastomer. The grip strength will depend on the changing stiffness value of the MR elastomer and weakened by the tensile force of the spring. However, the spring in this grip is necessary because it is involved in the regulation of the beam position. If capturing and holding an object is carried out by the right side of the gripper, then the gripping force will depend on the constant spring stiffness and will be weakened by the changing stiffness force of the MR elastomer. In general case, the maximum gripping force of both parts for the above gripper will be different, which confirms that for this approach the strength of a soft robot can be different depending on the application direction of the force.

If the varying stiffness force of the MR elastomer is compensated by the varying stiffness force of the other MR elastomer, then this approach can provide a uniformly large resulting force in any direction, however, the implementation of this approach requires a more complex control system. Errors in such control system can lead to the appearance of large values of mutually compensating stiffness forces of both elastomers, which can lead to damage to the soft robot.

The solution to the problems described earlier is not simple and depends on the requirements for its structure and the purposes of its application. For example, for solving the disadvantages of using varying stiffness force of the one MR elastomer to compensate for the varying stiffness force of another MR elastomer, increased requirements for the reliability of the control system are the most common solution. In addition, in some cases, it is possible to add additional elements to the system, which can affect it's work, but at the same time reduce the possibility of damage to individual elements of that system in case of an error in the control system. If these changes in the work of the system are within acceptable limits, then such a solution to the problem is also acceptable. Figure $2 \mathrm{c}$ ) shows an example of a two-link manipulator that can change the angular position between the two links using the varying stiffness forces of two MR elastomers. A high stiffness spring was added to this design as an additional element. If, as a result of a system error, a large stiffness force value for both MR elastomers occurs, then the additional spring will reduce the load on the individual elements of a two-link manipulator, which may be damaged.

Another advantage of using MR materials is the possibility of remote control of the soft robot using a magnetic field, which opens up great opportunities for reducing the number of elements in the softest robot and, as a result, its miniaturization. In [30], a snake-like robot is presented, which is capable of moving using a magnetic field. In this robot, instead of the effect of changing stiffness under the influence of a magnetic field, the ability of an anisotropic material to change its position under the influence of a magnetic field is used. Unlike the effect of changing stiffness, the robot can be controlled by changing not only the strength of the magnetic field, but also its direction.

A large space for research is devoted to the combination of several functions of one MR material in a soft robot [32]. MR elastomer can be used as part of sensor with compressive and magnetic sensing abilities. Also, MR elastomer can be used both as a sensor and as an actuator. Certain percentage of iron particles in MR elastomer could affect the electrical conductivity and be used to create force sensors. At the same time, its ability to change stiffness will be used to move the soft robot. However, this approach will definitely make its mark on the design of the soft robot. The selection of characteristics of the MR 
elastomer, acceptable for its use as a sensor, may negatively affect other characteristics that will be used to move the soft robot.

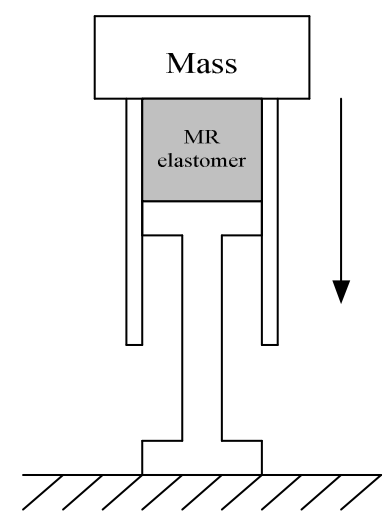

a)

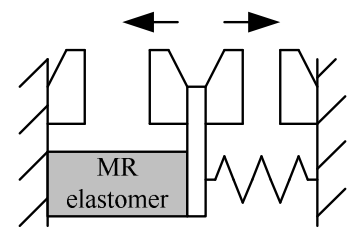

b)

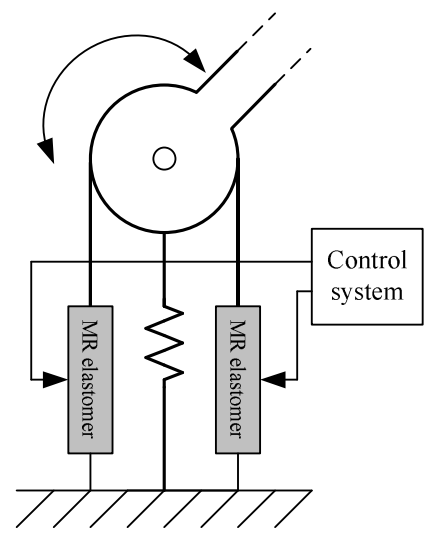

c)

Fig. 2. Examples of using MR elastomers in soft robots: a) a piston using MR elastomer, b) a gripper using MR elastomer, c) a two-link manipulator.

\section{Conclusions}

MR fluids and elastomers find more and more applications in various fields, including soft robots. Despite the fact that an increase in some useful characteristics can lead to a decrease in others, the correct selection of the characteristics of the MR material for a specific task can fully reveal its potential. The development of control systems allows for the creation of more complex, from the point of view of control, soft robots and, as a result, new approaches to their design. Therefore, the use of multiple MR elastomers to affect the movement of a soft robot may be a more acceptable solution. Search and research of new MR materials could expand the scope of their application, or make one and the same smart material based multifunctional within one soft robot.

\section{References}

1. S. Kim, C. Laschi, B. Trimmer, Trends Biotechnol. 31, 287-294 (2013)

2. A. Chen, R. Yin, L. Cao, C. Yuan, H. K. Ding, W. J. Zhang, 2017 24th International Conference on Mechatronics and Machine Vision in Practice, 366-370 (2017)

3. K. Kluszczyński, M. Kciuk, Int. J. Comput. Math. Electr. Electron. Eng. 32, 14171427 (2013)

4. Y. Nishioka, K. Nagao, S. Yasuda, T. Yasuda, M. Yamano, 2017 IEEE International Conference on Mechatronics and Automation, 1888-1893 (2017)

5. F. Jiang, Z. Zhang, X. Wang, G. Cheng, Z. Zhang, J. Ding, J. Intell. Robot. Syst. (2020)

6. E. Hasegawa, M. Hammadi, J.-Y. Choley, A. Ming, Design and Modeling of Mechanical Systems - IV Proceedings of the 8th Conference on Design and Modeling of Mechanical Systems, 882-891 (2020)

7. V. Skfivan, O. Sodomka, F. Mach, 2019 2nd IEEE International Conference on Soft Robotics, 126-130 (2019) 
8. N. S. Muhazeli, N. A. Nordin, S. A. Mazlan et al., J. Mater. Sci. 54, 13821-13833 (2019)

9. J. S. Kumar, P. S. Paul, G. Raghunathan, D. G. Alex, Int. J. Mech. Mater. Eng. 14, 13 (2019)

10. J. Y. Lee, S. H. Kwon, H. J. Choi, Korea-Australia Rheol. J. 31, 41-47 (2019)

11. M. R. Jolly, J. W. Bender, and J. D. Carlson, J. Intell. Mater. Syst. Struct. 10, 5-13 (1999)

12. A. Nedjar, S. Aguib, T. Djedid, A. Nour, A. Settet, M. Tourab, Silicon, 11, 1287-1293 (2019)

13. W. H. Li, Y. Zhou, T. F. Tian, Rheol. Acta, 49, 733-740 (2010)

14. M. A. Cantera, M. Behrooz, R. F. Gibson, F. Gordaninejad, Smart Mater. Struct. 26, 023001 (2017)

15. J. Gołdasz, S. Dzierżek, IOP Conf. Ser. Mater. Sci. Eng. 148, 012004 (2016)

16. L. Zhang, W. Wang, Y. Shi, Arab. J. Sci. Eng. 44, 2763-2773 (2019)

17. R. Jinaga, S. Kolekar, T. Jagadeesha, Automotive Tribology. Energy, Environment, and Sustainability, 237-268 (Springer, Singapore, 2019)

18. S. Kolekar, K. Venkatesh, J. Vib. Eng. Technol. 7, 107-116 (2019)

19. R. Brancati, G. Di Massa, S. Pagano, S. Santini, Meccanica, 54, 333-349 (2019)

20. V. P. Mikhailov, A. M. Bazinenkov, Instruments Exp. Tech. 59, 131-135 (2016)

21. W. H. Li, X. Z. Zhang, H. Du, Advances in Elastomers I. Advanced Structured Materials, 11, 357-374 (Springer, Berlin, Heidelberg, 2013)

22. Y. Li, J. Li, W. Li, H. Du, Smart Mater. Struct. 23, 123001 (2014)

23. H. Banerjee, H. Ren, Electromagnetic Actuation and Sensing in Medical Robotics. Series in BioEngineering, 43-72 (Springer, Singapore, 2018)

24. J. H. Kim, B. C. Kim, D. W. Lim, B.-C. Shin, J. Mech. Sci. Technol. 33, 5321-5325 (2019)

25. M. Lanzetta, K. Iagnemma, CIRP Ann. 62, 21-25 (2013)

26. X. Yin, S. Guo, Y. Song, Micromachines, 9, 465 (2018)

27. A. Pettersson, S. Davis, J. O. Gray, T. J. Dodd, T. Ohlsson, J. Food Eng. 98, 332-338, (2010)

28. Y. T. Choi, C. M. Hartzell, T. Leps, N. M. Wereley, AIP Adv. 8, 056701 (2018)

29. J. Cramer, M. Cramer, E. Demeester, K. Kellens, Procedia CIRP, 76, 127-132 (2018)

30. J. Kim, S. E. Chung, S.-E. Choi, H. Lee, J. Kim, S. Kwon, Nat. Mater. 10, 747-752 (2011)

31. Y. Yang, Y. Li, Y. Chen, Bio-Design Manuf. 1, 14-25 (2018)

32. T. Hu, S. Xuan, L. Ding, X. Gong, Mater. Des. 156, 528-537 (2018) 